This item was submitted to Loughborough's Research Repository by the author.

Items in Figshare are protected by copyright, with all rights reserved, unless otherwise indicated.

\title{
Influence of the molecular architecture on the adsorption onto solid surfaces:
} comb-like polymers

PLEASE CITE THE PUBLISHED VERSION

http://dx.doi.org/10.1039/c1cp21967g

PUBLISHER

(C) The Owner Societies / Royal Society of Chemistry

VERSION

AM (Accepted Manuscript)

LICENCE

CC BY-NC-ND 4.0

\section{REPOSITORY RECORD}

Guzman, Eduardo, Francisco Ortega, Margarita G. Prolongo, Victor Starov, and Ramon Rubio. 2011.

"Influence of the Molecular Architecture on the Adsorption onto Solid Surfaces: Comb-like Polymers". figshare. https://hdl.handle.net/2134/9228. 
This item was submitted to Loughborough's Institutional Repository (https://dspace.lboro.ac.uk/) by the author and is made available under the following Creative Commons Licence conditions.

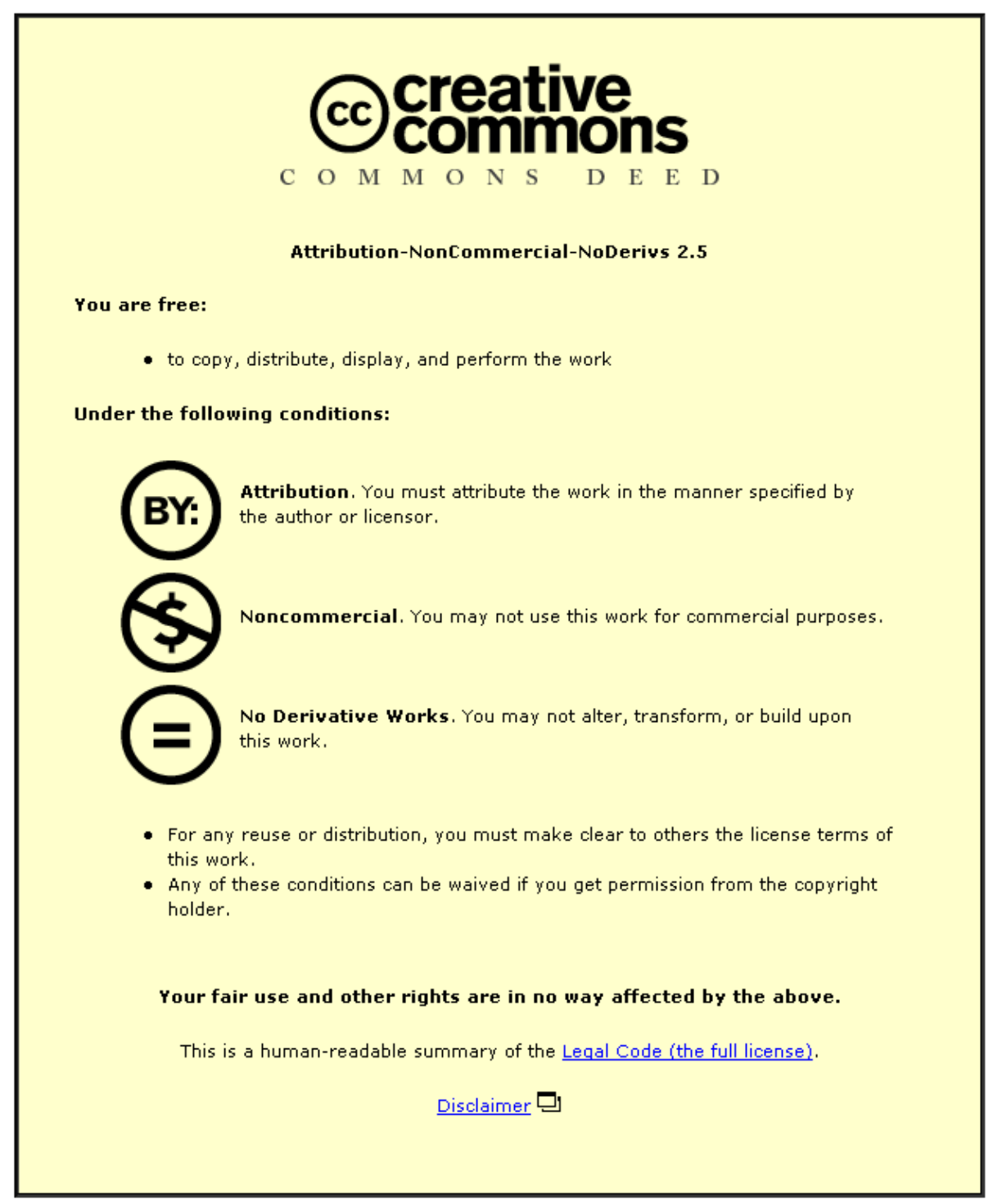

For the full text of this licence, please go to: http://creativecommons.org/licenses/by-nc-nd/2.5/ 
Cite this: Phys. Chem. Chem. Phys., 2011, 13, 16416-16423

\title{
Influence of the molecular architecture on the adsorption onto solid surfaces: comb-like polymers
}

\author{
Eduardo Guzmán, $\dagger^{* a}$ Francisco Ortega, ${ }^{a}$ Margarita G. Prolongo, ${ }^{b}$ \\ Victor M. Starov ${ }^{c}$ and Ramón G. Rubio ${ }^{* a}$ \\ Received 16th June 2011, Accepted 26th July 2011 \\ DOI: $10.1039 / \mathrm{c} 1 \mathrm{cp} 21967 \mathrm{~g}$
}

The processes of adsorption of grafted copolymers onto negatively charged surfaces were studied using a dissipative quartz crystal microbalance (D-QCM) and ellipsometry. The control parameters in the study of the adsorption are the existence or absence on the molecular architecture of grafted polyethyleneglycol (PEG) chains with different lengths and the chemical nature of the main chain, poly(allylamine) (PAH) or poly(L-lysine) (PLL). It was found out that the adsorption kinetics of the polymers showed a complex behavior. The total adsorbed amount depends on the architecture of the polymer chains (length of the PEG chains), on the polymer concentration and on the chemical nature of the main chain. The comparison of the thicknesses of the adsorbed layers obtained from D-QCM and from ellipsometry allowed calculation of the water content of the layers that is intimately related to the grafting length. The analysis of D-QCM results also provides information about the shear modulus of the layers, whose values have been found to be typical of a rubber-like polymer system. It is shown that the adsorption of polymers with a charged backbone is not driven exclusively by the electrostatic interactions, but the entropic contributions as a result of the trapping of water in the layer structure are of fundamental importance.

\section{Introduction}

The study of the adsorption of macromolecules (synthetic polymers, polyelectrolytes or proteins) onto solid ${ }^{1}$ (or fluid ${ }^{2}$ ) surfaces is a field that has had an intense development, both theoretically and experimentally, over the last years. ${ }^{3,4}$ This interest is due to the importance of polymer thin coating in many technological applications: fabrication of biocompatible films, stabilization of colloidal solutions and particle dispersions, flocculation process, surface treatment, cosmetics and, in general, in a large number of areas that are included in the field of nanotechnology. ${ }^{5}$

The modification of surfaces using comb-like polymers has received a great attention over the last years, ${ }^{6-8}$ because those polymers strongly modify the lubrication properties of the coated surfaces as revealed by macro- and microtribological studies. ${ }^{9-11}$ This is essentially due to the fluid character of the

\footnotetext{
${ }^{a}$ Departamento de Química Física I, Facultad de Ciencias Químicas, Universidad Complutense de Madrid, Ciudad Universitaria s/n, 28040-Madrid, Spain.E-mail: e.guzman@ge.ieni.cnr.it, rgrubio@quim.ucm.es

${ }^{b}$ Departamento de Materiales y Producción Aeroespacial, Universidad Politécnica, Ciudad Universitaria s/n, 28040-Madrid, Spain

${ }^{c}$ Chemical Engineering Department, Loughborough University, Loughborough, Leicestershire, LE11 3TU, UK

$\dagger$ Current address: CNR-Istituto per L'Energetica e le Interfasi, via Marini 6, 16149 Genova, Italy.
}

layer formed by the grafted chains that reduces the frictional forces. ${ }^{12}$ In addition comb-like polymers increase the solubility of complexes formed by polyelectrolytes and surfactants of opposite charges. ${ }^{13}$ These particular properties make comb-like polymers attractive for applications such as the fabrication of biomedical devices, ${ }^{14}$ or as conditioning agents in cosmetic applications. ${ }^{15}$ One of the most important advantages of the use of this type of comb-like polymers, in relation to other methods of fabrication of surfaces with grafted chains, is the possibility of tailoring the grafting density prior to the adsorption process. The latter allows selecting a polymer with the desired grafting ratio (ratio between the number of monomer units on the backbone and the number of grafted chains). ${ }^{11}$ High grafting ratios lead to the formation of surfaces without good tribological properties, whereas low grafting ratios result in not high enough binding to the surface. ${ }^{11,16}$

While the influence of the grafting ratio on the solution properties is well understood, less is known about its influence on interfacial properties such as adsorption and interfacial friction. Nanotribological studies of layers of PLL- $g$-PEG adsorbed onto silicon oxide substrates have shown that the adsorption of a grafted polymer on the surface reduces significantly the interfacial friction. ${ }^{9}$ Additionally, the investigation of PLL- $g$-PEG polymers that differ only in the length of the PEG side chains revealed that the interfacial forces measured in the aqueous medium decreased with an increase 
in the chain length of $\mathrm{PEG}^{9}{ }^{9}$ However, at the same time the lubrication properties were significantly improved ${ }^{10}$ as a consequence of the higher capacity of binding water by the longer PEG chains. ${ }^{17}$ More recently, Perry et al. ${ }^{18}$ have analyzed the effect of the adsorption time on the lubrication properties of PLL- $g$-PEG layers and showed that after 5 minutes of adsorption the lubrication properties are not modified considerably. However, publications on the adsorption kinetics and its relation to the final performance of the adsorbed films are very scarce.

The importance of the molecular architecture in the tribological properties of the surface modified by comb-like polymers is well known. ${ }^{16}$ Below we investigate the effect of the molecular architecture of grafted polymeric chains on several aspects of the adsorption process. We consider two types of possible modifications of the molecular architecture: (a) modifications of the length of the grafted PEG chains, and (b) modifications of the chemical nature of the main chain of the polymers, poly(allylamine) and poly(L-lysine). The adsorption kinetics, the total adsorbed amount, the water content, the shear modulus properties of the adsorbed films, and the mechanism driving the adsorption process are discussed, as well as the effect of polymer concentration on the adsorption.

\section{Experimental section}

\subsection{Chemicals}

Fig. 1 shows the chemical structure of the polymer used. We used three different polymers derived from polyallylamine. Linear-polyallylamine (PAH) was purchased from Aldrich (Germany) with a molecular weight of $17 \mathrm{kDa}$ and two different grafted copolymers of PAH and polyethyleneglycol (PEG) were purchased from Susos AG (Switzerland), where $\mathrm{PAH}$ of $14 \mathrm{kDa}$ is the main chain and the PEG chains appear to be grafted to the PAH chains with the grafting ratio of 3.5 in both cases. The difference between the two grafted polymers, G1 and G2, is the molecular weight of the PEG chains, $2 \mathrm{kDa}$ and $5 \mathrm{kDa}$, respectively. The influence of a chemical nature of the main chain was also investigated. For this purpose we used a poly(L-lysine) (PLL) based grafted copolymer purchased from Susos AG (Switzerland), where the main chain of PLL has a molecular weight of $20 \mathrm{kDa}$ with a grafting ratio of 3.5 and a molecular weight of the PEG chains is $5 \mathrm{kDa}$. The latter polymer is referred below as G3. All polymers were used as received without further purification.

Sodium chloride (purity $>99.9 \%$, Sigma-Aldrich (Germany)) was used for adjusting the ionic strength of the solutions to $100 \mathrm{mM}$ at $\mathrm{pH}=5.6$. The water used was of Milli-Q quality (Millipore RG model) with a resistivity higher than 18.2 M $\Omega$. All experiments were run at $(298.1 \pm 0.1) \mathrm{K}$. Solutions were prepared by weight using an analytical balance with a precision of $\pm 0.01 \mathrm{mg}$.

\subsection{Techniques}

A dissipative quartz-crystal microbalance (D-QCM) from KSV (Model QCM Z-500, Finland) was used. The quartz<smiles>CCC(C)CN</smiles>

(b)<smiles>COCCOCC(=O)NC(C)CC(C)(C)C</smiles>

(c)

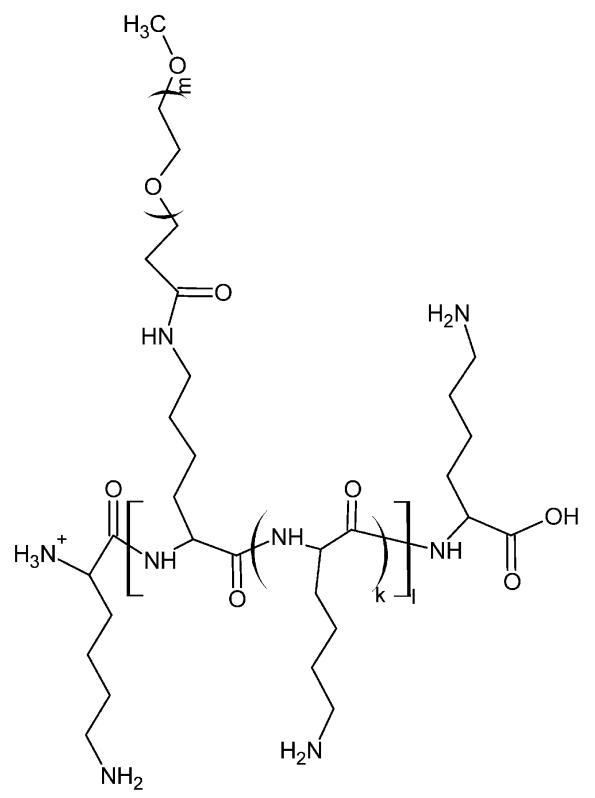

Fig. 1 Chemical structures of the polymers used in the study. (a) PAH. (b) PAH(14 kDa)-g(3.5)-PEG(2 or $5 \mathrm{kDa})$. (c) PLL(20 kDa)$g(3.5)-\operatorname{PEG}(5 \mathrm{kDa})$.

crystals, AT-cut, were cleaned with piranha solution $\left(70 \% \quad \mathrm{H}_{2} \mathrm{SO}_{4} / 30 \% \quad \mathrm{H}_{2} \mathrm{O}_{2}\right)$ over a period of thirty minutes and then thoroughly rinsed with pure water. The characteristic frequency under vacuum was $f_{0}=5 \mathrm{MHz}$. In order to charge the gold electrode of the quartz crystal, a self-assembled monolayer of sodium salt of 3-mercapto propanesulfonic acid was initially built onto the gold electrode, the surfaces modified in this way present a $\zeta$-potential value of $-42 \mathrm{mV}{ }^{19}$ D-QCM provided the impedance spectra of the crystal for the fundamental mode, and for odd overtones of the fundamental resonance up to the 11 th central frequency $\left(f_{11}=55 \mathrm{MHz}\right)$. For the analysis of the data, we adopted the model proposed by Johannsmann et al. ${ }^{20}$ that provides the total adsorbed mass (polymer and water) and the shear compliance of the adsorbed layer.

The polymer mass adsorbed has been measured by ellipsometry. We used an imaging null ellipsometer from Nanofilm (Model EP3, Germany). All the experiments were carried out using a solid-liquid cell at a fixed angle of $60^{\circ}$. Nature oxidized charged silicon wafers (Siltronix, France) were used as the substrates, the $\zeta$-potential of the silicon oxide was $-43 \mathrm{mV}$. The silicon wafers were cleaned following the same protocol as for the D-QCM quartz sensors. The ellipsometric angles $\Delta$ and $\Psi$ describe the changes in the state of polarization when light is reflected at a surface. ${ }^{21}$ For the analysis of the ellipsometry results, a four layer model has been used as in previous works. $^{22,23}$ 
We repeated some of the D-QCM experiments using an Au electrode coated with $\mathrm{SiO}_{2}$ in order to check whether the use of modified-gold or $\mathrm{SiO}_{2}$ surfaces had any significant influence on the adsorption. We found the same results as those with the thiolated gold electrode.

\section{Results and discussion}

\subsection{Adsorption process}

All the adsorption experiments were performed under static conditions without stirring. In Fig. 2 the results obtained by D-QCM for the adsorption process of a layer of G3 onto a solid substrate are shown as an example. Qualitatively similar results were found for all other polymers studied.

In Fig. $2 \mathrm{a}$ the shift in frequency of the quartz sensor, $\Delta f$, is presented. The shift was normalized by the number of overtones, $\nu$, for different overtones measured. In general overtones $\nu=3,5,7,9$ and 11 were measured, however, only $\nu=3,7$ and 11 are shown in Fig. 2a. Three consecutive stages can be distinguished as shown in Fig. 2a: first stage, the frequency of the base crystal immersed in the solvent was determined; second stage, the measuring chamber was then filled with the polymer solution, which resulted in a sharp decrease in $\Delta f / \nu$. The latter was due to the adsorption of material on the surface of the sensor. Note, possible changes in viscosity and density of the polymer solution were neglected because in dilute solutions those properties were virtually undistinguished from those of the solvent. Third stage: after the adsorption process was finished $(\Delta f / \nu=$ constant $)$ the measurement chamber was flushed with the solvent (rinsing process) in order to remove the chains which were weakly adsorbed on the substrate. Fast shifts in $\Delta f / \nu$ were observed during the injection and rinsing, however, they were not taken into account for the analysis of the adsorption process. ${ }^{24}$ The shift in the signal after the rinsing can be attributed to swelling/deswelling processes of the layers. The latter agree with the scenario of irreversible adsorption of grafted polymers reported previously by other authors. ${ }^{25,26}$

Fig. 2a also gives qualitative information on the mechanical behavior of the adsorbed polymer layers. For purely elastic layers (where the Sauerbrey equation is valid) ${ }^{20}$ the data $\Delta f / \nu$ of all the overtones should collapse onto a single master curve. This was not the case in our experiments, which indicates that the adsorbed layers behave as viscoelastic layers. ${ }^{20}$ The latter was further confirmed by an increase in the dissipation factor, $D$, presented in Fig. 2b. Note in Fig. 2b each experimental point corresponds to a different time. Fig. $2 b$ shows that the dissipation factor $D$ sharply increased during the adsorption process after a short induction period. Data presented in Fig. $2 b$ give additional information on the existence of rearrangement of the preadsorbed chains, which is related to the type of behaviour of the plot (linear or non-linear trends). ${ }^{27}$ Fig. 2 b also allows obtaining qualitative information on energy/entropy balance in the adsorption process. ${ }^{28}$ High $\Delta D$ values in this plot indicate that the energetic factors predominate, while low $\Delta D$ values indicate that entropy is the factor that controls the adsorption process. The results obtained by ellipsometry are in good qualitative agreement with those obtained from D-QCM and they are in agreement with the results obtained for other similar systems. ${ }^{15,29}$

\subsection{Adsorption kinetics}

The adsorption kinetics is controlled by a complex balance of polymer-solvent and polymer-surface interactions. ${ }^{30}$ There are several theoretical studies in the literature available on this topic. ${ }^{31-33}$ However, there are substantial problems in studying the kinetics of the process experimentally in a very short time scale. ${ }^{34-36}$ The kinetic data presented below can be described as those from a bimodal process in the way proposed by Chiang et al. ${ }^{37}$ Our data agree with the results previously reported for other polyelectrolyte systems. ${ }^{15}$ The adsorption process proceeds in two steps. The first fast step can be related to the transport of the chains to the surface. However, it was found that the mentioned transport process was not diffusion controlled because the adsorbed mass did not show $a t^{1 / 2}$ dependence. The latter conclusion is in agreement with the predictions made by Cohen-Stuart ${ }^{31}$ and could be related to the first two stages according to the model proposed by Linse and Källrot ${ }^{33}$ for the adsorption of neutral polymers. After the
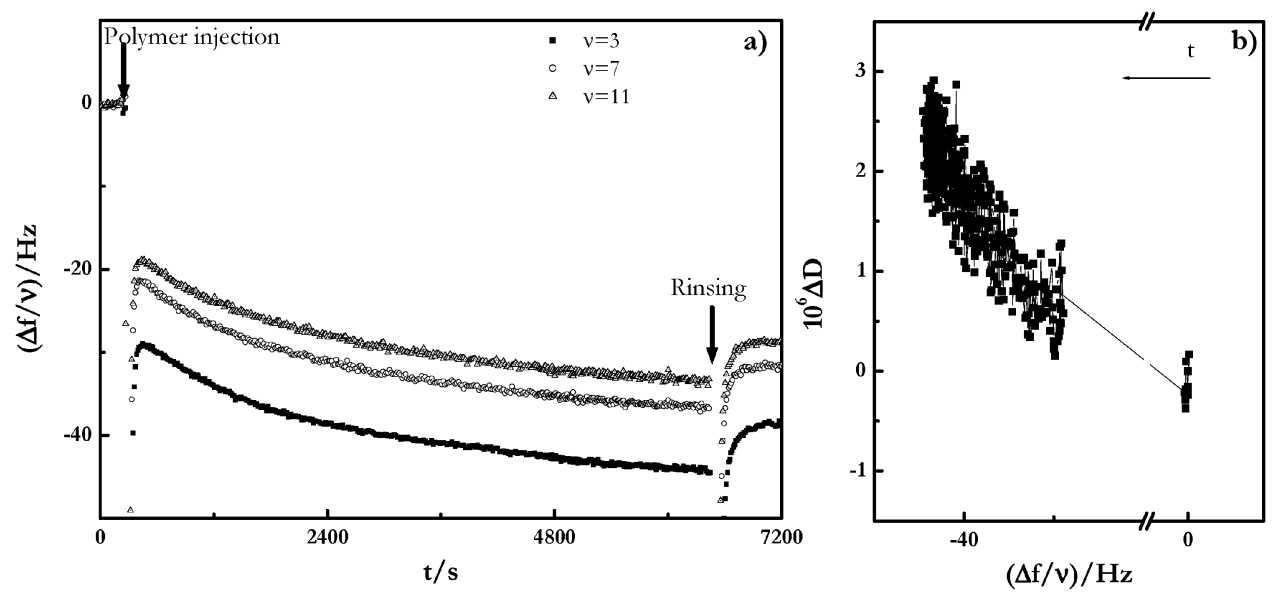

Fig. 2 (a) Kinetics of the adsorption and rinsing for the adsorption of a layer of G3: the time variation of the shift of the central frequency of the different overtones of the quart sensors. For clarity only the curves for $\nu=3,7$ and 11 are shown. (b) $\Delta D v s$. $\Delta f / \nu$ graph. The experiments correspond to a polymer solution with $c \approx 0.05 \mathrm{mg} \mathrm{mL}^{-1}$. 


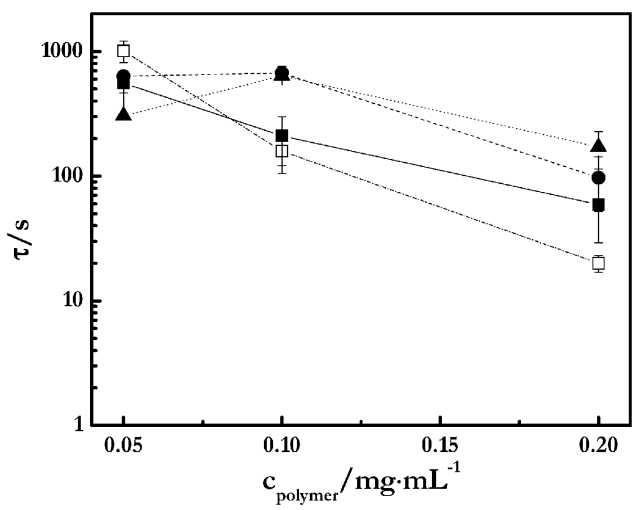

Fig. 3 Adsorption times of different studied polymers. Legend: $\mathbf{\square}-$ PAH; • - - , G1; $\mathbf{\Delta} \cdots, \mathrm{G} 2 ; \square$-.-, G3.

first step almost $70-80 \%$ of the total adsorption was reached. The second slow step might be related to the reorganization of the preadsorbed chains. This model agrees with the description by Perry et al. ${ }^{18}$ on the adsorption of PLL- $g$-PEG copolymers. However, below only the total adsorption time, $\tau$, required for the complete adsorption of the polymers used is presented.

In Fig. 3 the values of $\tau$ are summarized for different polymers studied. The $\tau$ values decrease as the polymer concentration increases for all the polymers used as expected. The data presented in Fig. 3 also demonstrate a slowing down of the adsorption kinetics with the increase in the size of the grafted chains. The slowing down can be due to the steric limitations imposed by the grafted chains which restricts the reorganization process. The reorganization is expected to be more important for longer PEG grafted chains (recall that in this case the total molecular weight is larger). ${ }^{8}$ The change of PAH to PLL as the main chain results in a faster adsorption kinetics. The latter can be attributed to a higher affinity of the PLL to the surface. The higher separation of PEG grafted chains in PLL-based copolymers as compared with PAH-based copolymers at the same grafting ratio is likely responsible for this. Note, the monomer sizes are 2.8 and $3.6 \AA$ for PAH and PLL, respectively. As a consequence the steric repulsion between PEG chains with a weaker interaction with the surface makes the adsorption easier for the copolymer with PLL. A similar explanation has been also proposed by Hartung et al. ${ }^{16}$ as a characteristic of the interaction between amine based polymers and negatively charged surfaces.

\subsection{Adsorbed mass and water content}

The data in Fig. 4 summarize the amount of material adsorbed. These data were obtained using both the D-QCM technique, $\Gamma_{\text {ac }}$, and ellipsometry, $\Gamma_{\text {op }}$, for the different layers studied. The amount of adsorbed material obtained using the D-QCM is higher than that using ellipsometry in all the cases studied. The latter is due to the different sensitivity of those methods to the adsorbed amount. ${ }^{38}$ The total thickness of the layers increased with the concentration of polymers for all the polymers studied as expected. The latter can be understood by considering the arrangement in the layers of the adsorbed polymer chains: for low polymer concentration the polymer chains adsorb on the surface in an extended conformation; however, more chains compete for the adsorption sites on the
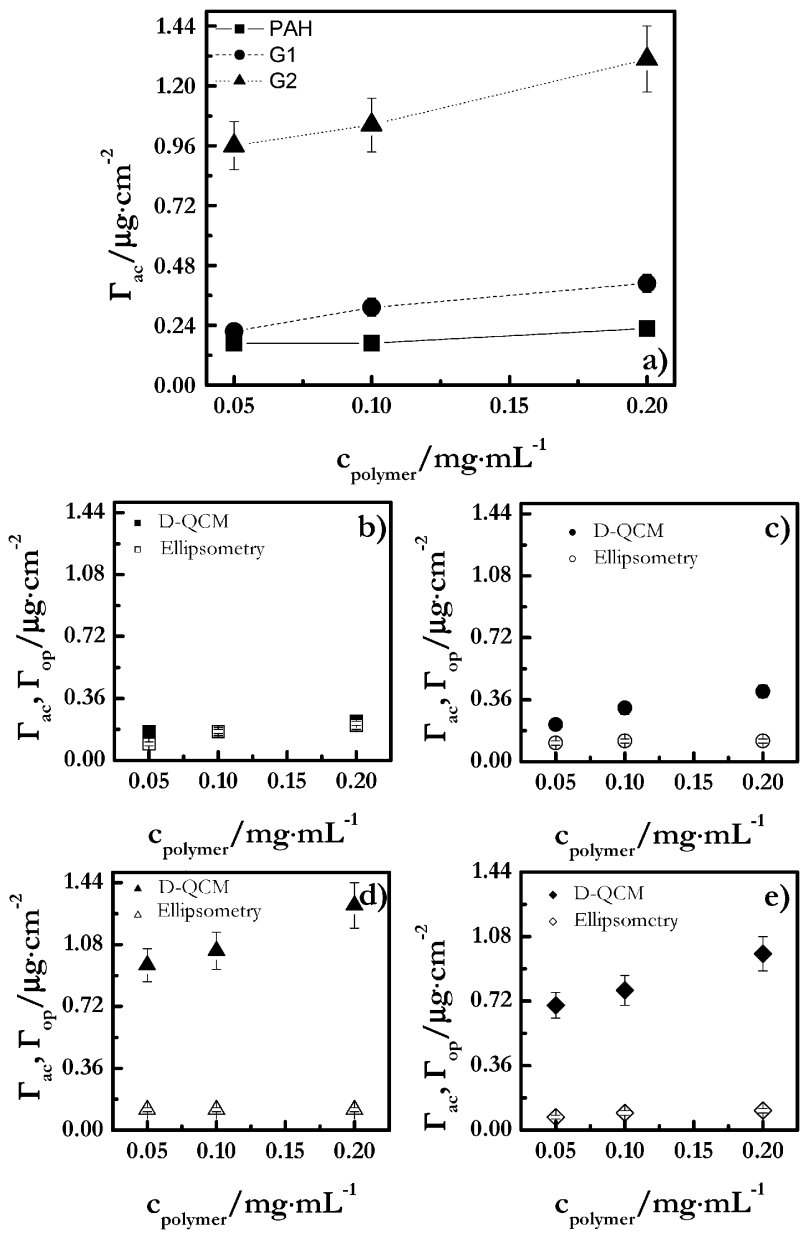

Fig. 4 (a) Experimental isotherm obtained using D-QCM (symbols) for the different poly(allylamine) based polymers studied. From (b) to (e): comparison of the experimental isotherm obtained using D-QCM and ellipsometry for the different polymers: (b) PAH, (c) G1, (d) G2, (e) G3.

surface at higher concentrations. The latter results in an increasing disorder of the adsorbed layers with an increase in the number of segments protruding into the solution, and a higher adsorbed mass as a result. The adsorbed mass increases with the size of the grafted chains, it is expected that the PEG chains are expanded into the solution as water is a good solvent. ${ }^{39}$ The latter conclusion is in agreement with the results for PLL- $g$-PEG by Perry et al. ${ }^{18}$ and Müller et al. ${ }^{26}$ Our results show that the adsorption is not governed solely by the length of the grafted chains, but also by the chemical nature of the main chain as shown in Fig. 4d and e. The latter figures show that the adsorption of $\mathrm{G} 2$ is higher than that of $\mathrm{G} 3$, while the length of the grafted chains is identical in both cases.

It is possible to extract the relative packing of the grafted chains in the film using the results obtained by ellipsometry. The efficiency of the surface grafting is defined by the areal density of PEG chains $n_{\mathrm{PEG}}{ }^{39}$ (see Fig. 5a):

$$
n_{\mathrm{PEG}}=\frac{\Gamma_{\mathrm{op}} N_{\mathrm{A}}}{M_{\text {backbone }} g+M_{\mathrm{PEG}}},
$$

where $\Gamma_{\text {op }}$ is the adsorbed mass per unit area measured by ellipsometry, $M_{\text {backbone }}$ is the molecular weight of one 

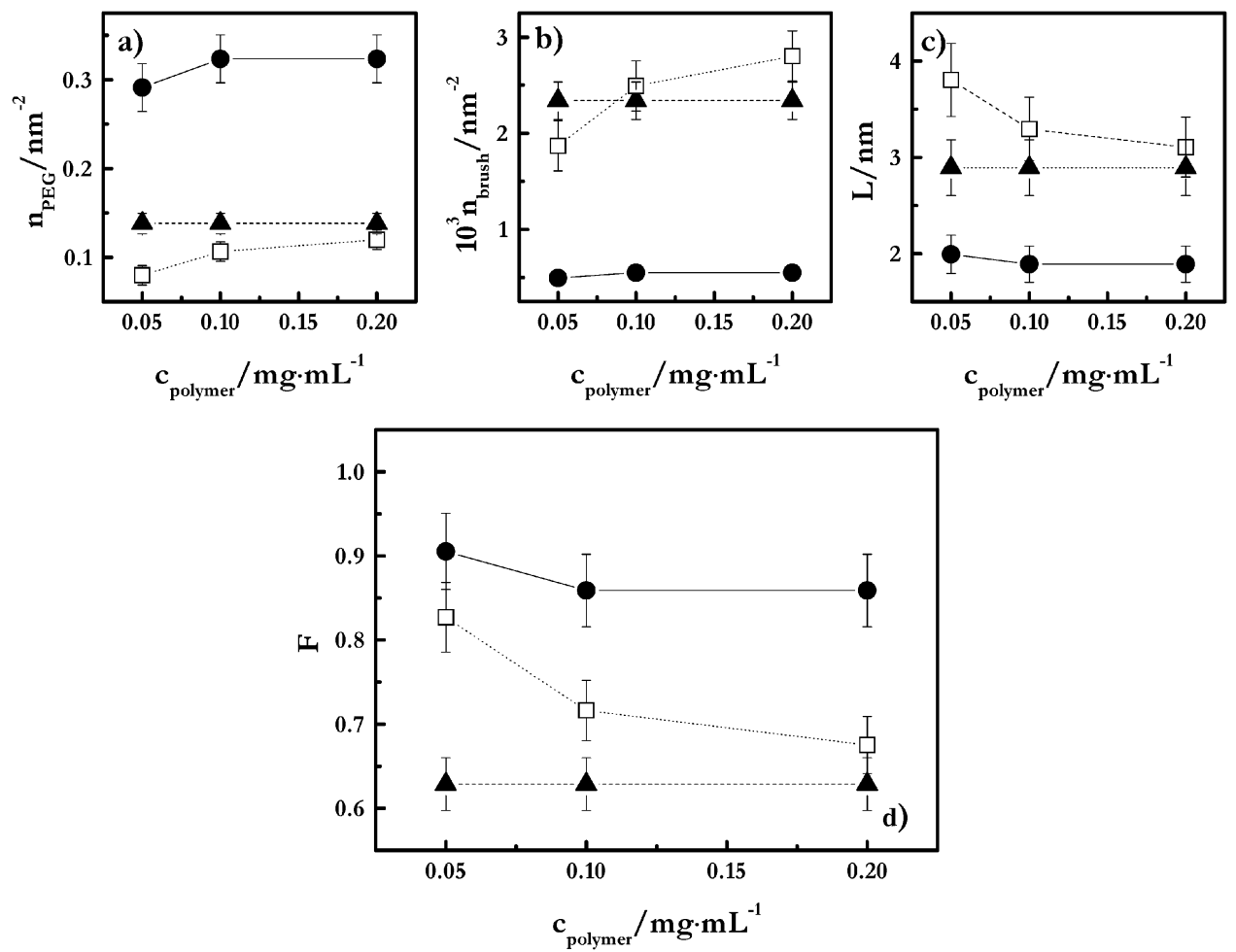

Fig. 5 For grafted chains (G1, G2 and G3). (a) $n_{\mathrm{PEG}} v s$. concentration of polymers. (b) $n_{\text {brush }} v s$. concentration of polymers. (c) $L$ vs. concentration of polymers. (d) Relative packing factor $(F) v s$. concentration of polymers. The lines show the average trend of the data. Legend: $\bullet-., \mathrm{G} 1 ; \boldsymbol{\Delta} \cdots, \mathrm{G} 2 ; \square--, \mathrm{G} 3$.

backbone repeat unit (not including the counterion), $g$ represents the grafting ratio, $M_{\mathrm{PEG}}$ is the molecular weight of one PEG side chain, and $N_{\mathrm{A}}$ is Avogadro's number. It is possible to define the surface density of copolymer molecules, $n_{\text {brush }}$, in a similar way. $n_{\text {brush }}$ was calculated in the following way:

$$
n_{\text {brush }}=\frac{\Gamma_{\mathrm{op}} N_{\mathrm{A}}}{M_{\mathrm{brush}}},
$$

that is an expression similar to eqn (1) (see Fig. 5b). In the latter equation $M_{\text {brush }}$ is the molecular weight of the copolymer. The estimated distance between PEG side chains along the backbone may be calculated as the grafting ratio multiplied by the length of the backbone's repeating unit, ${ }^{16}$ that is $9.8 \AA$ and $12.6 \AA$ for PAH-based and PLL-based grafted copolymers, respectively. The relative packing factor, $F$, is defined as $F=L / 2 R_{\mathrm{g}},{ }^{25,39}$ where $R_{\mathrm{g}}$ is the radius of gyration of the PEG chains and $L$ is the average spacing between the PEG side chains. $F$ describes the degree of overlapping between the PEG chains: a low value of the relative packing factor means a high degree of overlapping between the PEG side-chains that extend from the surface as a result of the steric effects. $^{11}$

The calculation of $R_{\mathrm{g}}$ and $L$ considers the existence of backbones that are extended parallel to the surface and $R_{\mathrm{g}}$ can be calculated using an empirical formula based on light scattering measurements: ${ }^{40}$

$$
R_{\mathrm{g}}=0.181 N^{0.58}(\mathrm{~nm}),
$$

where $N$ is the number of ethlyleneglycol monomers on the PEG chains. This leads to values of $R_{\mathrm{g}}$ of 1.1 and $2.3 \mathrm{~nm}$ for
PEG chains of $2 \mathrm{kDa}$ and $5 \mathrm{kDa}$, respectively. $L$ is calculated as $^{39}$ (see Fig. 5c):

$$
L=\left(\frac{2}{\sqrt{3} n_{\mathrm{PEG}}}\right)^{0.5}
$$

The results presented in Fig. 5 show that the packing of the grafted chains is controlled by both the size of the grafted chains and the chemical nature of the main chain. The contribution of the length of the grafted chains may be attributed to the existence of a higher influence of steric hindrance: the PEG chain length increases leading to a decrease of the packing of the chains. The contribution of the molecular nature of the main chain may be due to the distance between monomers of PLL and PAH: for identical grafting ratios and lengths of the PEG, in the case of G2, the PEG chains are closer thus increasing the importance of the steric contribution and leading to a lower packing in $\mathrm{G} 2$ than in G3. The decrease in the packing density with an increase in the concentration may be due to the higher mass adsorbed that can increase the steric effects. ${ }^{16}$ The packing of the PEG chains is strongly correlated with the water content inside the adsorbed layer. It is important to remind that the charged polymer chains adsorb in a hydrated form which explains why in all the cases $\Gamma_{\text {ac }}>\Gamma_{\text {op }}{ }^{26,38}$ The weight fraction of water associated with the adsorbed layer, $X_{\mathrm{w}}$, can be obtained by comparing the adsorbed amount from D-QCM measurements, acoustical mass, $\Gamma_{\text {ac }}$, and ellipsometry, $\Gamma_{\text {op }}$, using the procedure introduced by Vörös. ${ }^{38}$ The latter procedure gives values 


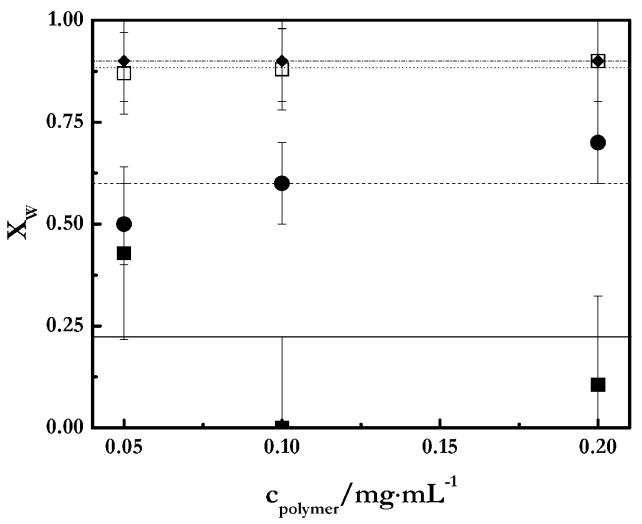

Fig. 6 Water content for the layers of the different polymers studied. The lines show the average trend of the data. Legend: $\mathbf{\square}-, \mathrm{PAH}$; - - - , G1; $\mathbf{\Delta} \cdots, \mathrm{G} 2 ; \square$-.-, G3.

of $X_{\mathrm{w}}$, which correlate with those obtained using neutron reflectivity: ${ }^{19}$

$$
X_{\mathrm{w}}=\frac{\Gamma_{\mathrm{ac}}-\Gamma_{\mathrm{op}}}{\Gamma_{\mathrm{ac}}},
$$

The differences between acoustical and optical masses are shown in Fig. 4b-e for the different polymers. The water contents reported in Fig. 6 show a clear dependence on the length of the grafted chains, while there is no dependence on the chemical nature of the main chain. This is because most of the water hydrated the PEG chains that protrude into the solution. Hence, the increase in the adsorbed amount of the layer also increases the water content of the layers ${ }^{39}$ in agreement with the decrease in the packing density of the grafted chains.

From the results, it is possible to estimate the average number of solvent molecules for polyethyleneglycol chains, $\Lambda_{\mathrm{EG}}$, adapting the method proposed by Müller et al.: ${ }^{26}$

$$
\begin{gathered}
\Sigma_{\mathrm{H}_{2} \mathrm{O}}=\frac{N_{\mathrm{A}}\left(\Gamma_{\mathrm{ac}}-\Gamma_{\mathrm{op}}\right)}{M_{\mathrm{H}_{2} \mathrm{O}}}, \\
\Sigma_{\mathrm{EG}}=\frac{N_{\mathrm{A}} M_{\mathrm{PEG}} \Gamma_{\mathrm{op}}}{M_{\mathrm{EG}}\left(M_{\mathrm{monomer}} g+M_{\mathrm{PEG}}\right)}, \\
\Lambda_{\mathrm{EG}}=\frac{\Sigma_{\mathrm{H}_{2} \mathrm{O}}}{\sum_{\mathrm{EG}}},
\end{gathered}
$$

where $\Sigma_{\mathrm{H}_{2} \mathrm{O}}$ and $\Sigma_{\mathrm{EG}}$ are the surface densities of the solvent and the ethyleneglycol monomers, respectively; $M_{\mathrm{H}_{2} \mathrm{O}}, M_{\mathrm{EG}}$ and $M_{\text {monomer }}$ are the molecular weights of water, ethyleneglycol and monomer units of the main chain of the grafted copolymer, respectively. The results for $\Lambda_{\mathrm{EG}}$ for the different layers analyzed are shown in Fig. 7. The number of molecules of water for monomeric units of ethyleneglycol is in good agreement with the values of $F$ for the grafted chains previously discussed, and with the values of water content obtained for the different layers studied.

\subsection{Mechanical properties}

The analysis of D-QCM experimental data provides information on the mechanical behavior of the adsorbed layers. The complex compliance $\hat{J}(f)$ allows calculating the shear modulus

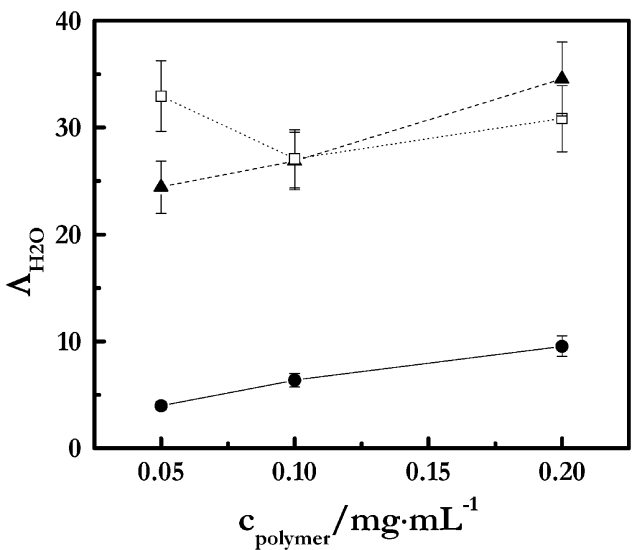

Fig. 7 Number of water molecules for ethyleneglycol monomer units for the grafted chains. The lines show the average trend of the data. Legend: •, G1; $\boldsymbol{\Delta}, \mathrm{G} 2 ; \square, \mathrm{G} 3$.
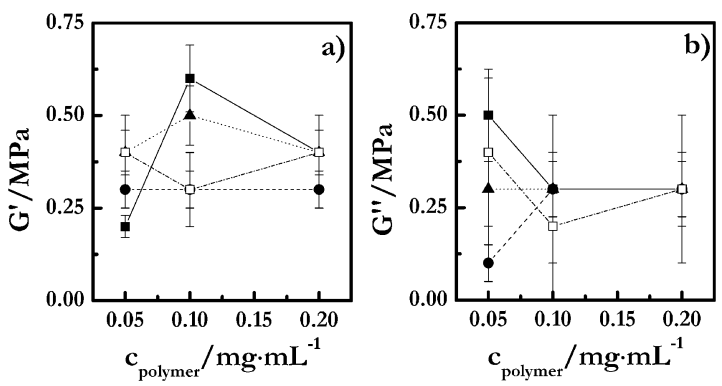

Fig. 8 (a) $G^{\prime}$ vs. $c_{\text {polymer }}$ (b) $G^{\prime \prime}$ vs. $c_{\text {polymer }}$ Legend: $\mathbf{\square}-$, PAH; •- - - , G1; $\mathbf{\Delta} \cdots, \mathrm{G} 2 ; \square$-.-, G3.

$\hat{G}(f)=1 / \hat{J}(f)$, and hence its real, $G^{\prime}$, and imaginary components, $G^{\prime \prime} .{ }^{20}$ The results for all the systems studied are in the range of MPa (Fig. 8), typical for highly plasticized polymers as a consequence of the associated water. ${ }^{18,41}$ For the systems investigated $G^{\prime} \approx G^{\prime \prime}$ that is typical for gel-like materials.

\subsection{Control of the adsorption process: entropic vs. energetic contributions}

The entropic contribution to the adsorption process was studied using D-QCM. ${ }^{42}$ For this purpose, we plotted the shift of the dissipation factor for the third overtone of the quartz sensor versus the change in the normalized frequency for the same overtone (the results for the other overtones were similar). Olanya et al. $^{43}$ have pointed out that an increase in the dissipation factor is associated with a lower entropic contribution. This assumption was also used by Ozeki et $\mathrm{al}^{44}$ in the analysis of the binding processes of different biopolymers to the quartz crystal sensor. Fig. 9 shows the effect of the size of the grafted chains of PAH based copolymers on the entropic contribution of the adsorption process. The latter is presented as the variation of the dissipation factor, $\Delta D$, for the third overtone, with the change in the normalized frequency for the same overtone. Note that the entropic/energetic contributions here obtained are established in a relative scale, ${ }^{45}$ thus they are only useful for the comparison of the layers of the different polymers when they present 


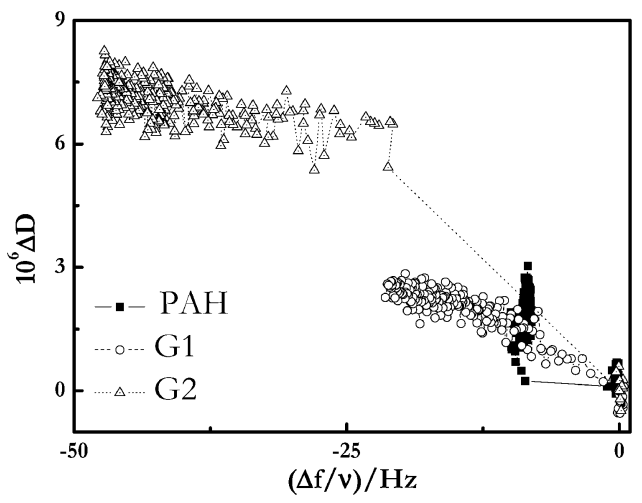

Fig. 9 Effect of the size of the grafted chains on the entropic contribution to the adsorption process represented as the shift in dissipation factor of the third overtone versus shift in normalized frequency for the adsorption of the different poly(allylamine) based polymers studied from solutions of concentration $0.1 \mathrm{mg} \mathrm{mL}^{-1}$.

similar lengths of the PEG-grafted chains, similar chemical nature of the main chain or both simultaneously.

The results presented in Fig. 9 prove that the entropic contribution to the adsorption process strongly depends on the size of the grafted chains. The increase in the length of the grafted chains decreases the entropic contribution (increase in the absolute value of the entropy) and enhances the energetic contribution (increase in the absolute value of the energy) to the adsorption. This counterintuitive behavior can be explained by the formation of an ordered liquid layer around the grafted chain: it has been already mentioned that longer grafted chains promote the formation of thicker ordered liquid layers in accordance with the higher values of $\Gamma_{\mathrm{ac}}$ (Fig. 4) and with the higher water content (Fig. 6). Note, for the PAH and G1 the effect on the entropic contribution is similar and smaller than for G2. The latter suggests that a minimum value of the grafted chain length is required to obtain an important effect on the entropic contributions to the adsorption. This may result in the improving of the lubrication properties as the length of the grafted chains increases due to the increase in the water content of the layers (Fig. 6). ${ }^{18}$ The results presented in Fig. 10a confirm the above assumption: the adsorption is controlled by the energetic effect independently of the molecular nature of the main chain (G2 and G3), which agrees with the fact that the water content in both cases is very similar (Fig. 6). The smaller differences observed between G2 and G3 may be due to the effect of the structure of the main chain where the higher flexibility of the amine groups of PLL increases the entropic contribution. ${ }^{16,33}$ The increase on the number of segments protruding into the solution with polymer concentration also increases the water trapped by the adsorbed layer, thus reducing the entropic contribution (increase in the absolute value of the entropy) as shown in Fig. 10b for G1 (similar results were found for G2 and G3).

\section{Conclusions}

The adsorption of grafted polymers was studied using both D-QCM and ellipsometry. The influence of polymer concentration, the length of the PEG grafted chains and the chemical
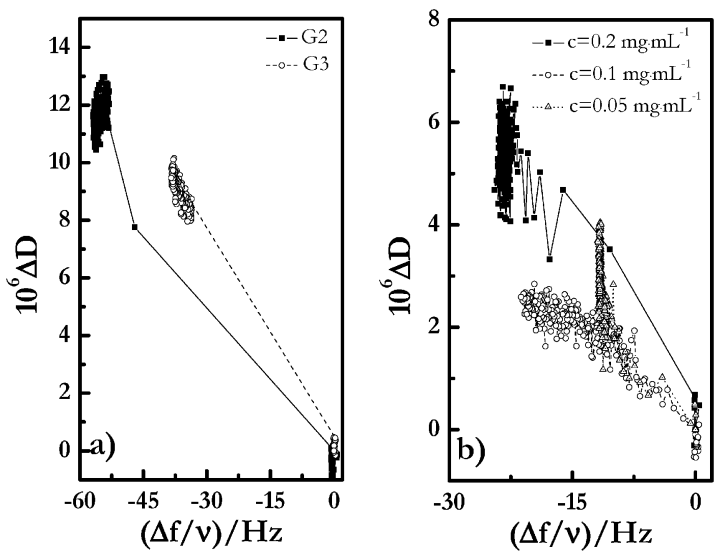

Fig. 10 Effect of the polymer concentration on the entropic contribution to the adsorption process represented as the shift in the dissipation factor of the third overtone versus shift in the normalized frequency for the adsorption of the different layer. (a) Influence of the nature of the main chain on the entropic contribution for layers of G2 and $\mathrm{G} 3$ adsorbed from solution of $c_{\text {polymer }}=0.2 \mathrm{mg} \mathrm{mL}^{-1}$. (b) Example of the effect of the polymer concentration on G1 layers.

nature of the main chains are discussed. Both techniques indicate that the adsorption process was essentially irreversible on the experimental time scale used. It is shown that the adsorption kinetics of the polymers investigated is a complex process which involves several steps. The adsorbed amount of polymer depends on the polymer concentration, the length of the grafted chains and the chemical nature of the main chain. The comparison of the thicknesses of the adsorbed polymer layers obtained by D-QCM and ellipsometry allowed estimation of the water content inside the adsorbed layers, which showed dependence on the length of the grafted chains. The D-QCM allowed calculation of the complex shear modulus of the adsorbed polymer layers, $G^{\prime}$ and $G^{\prime \prime}$, at low frequencies. The latter had values in the range of a rubber-like polymer. The entropic contribution associated with the adsorption is related to the trapping of water molecules around the grafted chains.

\section{Acknowledgements}

This work was supported in part by MICINN under grant FIS2009-14008-C02-01, by ESA under grant MAP AO-00-052 (FASES) and PASTA, and by UE under ITN MULTIFLOW. E.G. was supported by a FPU fellowship from MICINN. The authors are grateful to the UIRC of the CAI of Spectroscopy of Complutense University for the use of the ellipsometer.

\section{References}

1 K. Holmberg, B. Jönsson, B. Kronberg and B. Lindman, Surfactants and Polymers in Aqueous Solution, John Wiley \& Sons, Chichester, 2002.

2 E. Guzmán, H. Ritacco, F. Ortega, T. Svitova, C. J. Radke and R. G. Rubio, J. Phys. Chem. B, 2009, 113, 7128-7137.

3 R. R. Netz and D. Andelman, Phys. Rep., 2003, 380, 1-95.

4 G. J. Fleer, M. A. Cohen Stuart, J. M. H. M. Scheujtens, T. Cosgrove and B. Vicent, Polymer at Interfaces, Chapman\&Hall, Cambridge, 1993.

5 J. A. Hubbell, Curr. Opin. Biotechnol., 1999, 10, 123-129.

6 S. Lee and N. D. Spencer, Science, 2008, 319, 575-576. 
7 B. Liberelle and S. Giasson, Langmuir, 2008, 24, 1550-1559.

8 L. Feuz, F. A. M. Leermakers, M. Textor and O. Borisov, Langmuir, 2008, 24, 7232-7244.

9 X. Yan, S. S. Perry, N. D. Spencer, S. Pasche, S. M. De Paul, M. Textor and M. S. Lim, Langmuir, 2004, 20, 423-428.

10 S. Lee, M. Müller, M. Ratoi-Salagean, J. Vörös, S. Pasche, S. D. De Paul, H. A. Spikes, M. Textor and N. D. Spencer, Tribol. Lett., 2003, 15, 231-239.

11 M. Müller, S. Lee, H. A. Spikes and N. D. Spencer, Tribol. Lett., 2003, 15, 395-405.

12 J. Klein, E. Kumacheva, D. Mahalu, D. Perahia and L. J. Fetters, Nature, 1994, 370, 634-636.

13 A. V. Svensson, L. Huang, E. S. Johnson, T. Nylander and L. Piculell, ACS Appl. Mater. Interfaces, 2009, 1, 2431-2442.

14 Y. Uyama, H. Tadokoro and Y. Ikada, Biomaterials, 1991, 12, $71-75$.

15 E. Guzmán, F. Ortega, N. Baghdadli, G. S. Luengo and R. G. Rubio, Colloids Surf., A, 2011, 375, 209-218.

16 W. Hartung, T. Drobek, S. Lee, S. Zürcher and N. D. Spencer, Tribol. Lett., 2008, 31, 119-128.

17 T. Drobek and N. D. Spencer, Langmuir, 2008, 24, 1484-1488.

18 S. S. Perry, X. Yan, F. T. Limpoco, S. Lee, M. Müller and N. D. Spencer, ACS Appl. Mater. Interfaces, 2009, 6, 1224-1230.

19 E. Guzmán, H. Ritacco, J. E. F. Rubio, R. G. Rubio and F. Ortega, Soft Matter, 2009, 5, 2130-2142.

20 D. Johannsmann, K. Mathauer, G. Wegner and W. Knoll, Phys. Rev. B: Condens. Matter, 1992, 46, 7808-7815.

21 R. M. A. Azzam and N. M. Bashara, Ellipsometry and Polarized Light, Elsevier, North-Holland, 1987.

22 E. Guzmán, F. Ortega, M. G. Prolongo, M. A. Rubio and R. G. Rubio, J. Mater. Sci. Eng., 2010, 4, 1-13.

23 E. Guzmán, V. San Miguel, C. Peinado, F. Ortega and R. G. Rubio, Langmuir, 2010, 26, 11494-11502.

24 T. Viitala, J. T. Hautala, J. Vuorinen and S. K. Wiedmer, Langmuir, 2007, 23, 609-618.

25 G. L. Kenausis, J. Vörös, D. L. Elbert, N. Huang, R. Hofer, L. Ruiz-Taylor, M. Textor, J. A. Hubbell and N. D. Spencer, J. Phys. Chem. B, 2000, 104, 3298-3309.
26 M. T. Müller, X. Yan, S. Lee, S. S. Perry and N. D. Spencer, Macromolecules, 2005, 38, 5706-5713.

27 M. R. Nejadnik, A. L. J. Olsson, P. K. Sharma, H. C. van der Mei, W. Norde and H. J. Busscher, Langmuir, 2009, 25, 6245-6249.

28 E. Gurak, C. Dupont-Gillain, J. Booth, C. J. Roberts and P. G. Rouxhet, Langmuir, 2005, 21, 10684-10692.

29 E. Guzmán, J. A. Cavallo, R. Chuliá-Jordán, C. Gómez, M. C. Strumia, F. Ortega and R. G. Rubio, Langmuir, 2011, 27, $7128-7137$.

30 L.-C. Jia and P.-Y. Lai, J. Chem. Phys., 1996, 105, 11319-11325.

31 M. A. Cohen-Stuart, J. Phys.: Condens. Matter, 1997, 9, 7767-7783.

32 N. Källrot, M. Dahlqvist and P. Linse, Macromolecules, 2009, 42, 3641-3649.

33 P. Linse and N. Källrot, Macromolecules, 2010, 43, 2054-2068.

34 T. J. Lane, W. R. Fletcher, M. V. Gormally and M. S. Johal, Langmuir, 2008, 24, 10633-10636.

35 G. Bijelic, A. Shovsky, I. Varga, R. Makuska and P. M. Claesson, J. Colloid Interface Sci., 2010, 348, 189-197.

36 E. Guzmán, H. Ritacco, F. Ortega and R. G. Rubio, Colloids Surf., A, 2011, 384, 274-281.

37 C.-Y. Chiang, V. M. Starov and D. R. Lloyd, Colloid J. (Transl. of Kolloidn. Zh.), 1995, 57, 715-724.

38 J. Vörös, Biophys. J., 2004, 87, 553-561.

39 S. Pasche, S. M. De Paul, J. Vörös, N. D. Spencer and M. Textor, Langmuir, 2003, 19, 9216-9225.

40 S. Kawaguchi, G. Imai, J. Suzuki, A. Miyahara, T. Kitano and K. Ito, Polymer, 1997, 38, 2885-2891.

41 R. G. Larsson, The Structure and Rheology of Complex Fluids, Oxford University Press, Oxford, 1999.

42 F. Höok, M. Rodahl, P. Brzezinski and B. Kasemo, Langmuir, 1998, 14, 729-734.

43 G. Olanya, J. Iruthayaraj, E. Poptoshev, R. Makuska, A. Vareikis and P. M. Claesson, Langmuir, 2008, 24, 5341-5349.

44 T. Ozeki, M. Morita, H. Yoshimine, H. Furusawa and Y. Okahata, Anal. Chem., 2007, 79, 79-88.

45 E. Guzmán, F. Ortega, N. Baghdadli, C. Cazeneuve, G. S. Luengo and R. G. Rubio, ACS Appl. Mater. Interfaces, 2011, DOI: $10.1021 / \mathrm{am} 200671 \mathrm{~m}$, in press. 